\title{
LONG-REACH OPTICAL ACCESS NETWORKS (LR-OANS): A PROMISING CANDIDATE FOR FUTURE OPTICAL ACCESS
}

\author{
Mohamed I.M.M. and M.S. Ab-Rahman \\ Department of Electrical, Electronics and System Engineering, \\ UKM University, Bangi, Malaysia
}

Received 2014-02-19; Revised 2014-02-20; Accepted 2014-08-12

\begin{abstract}
The ever-increasing demand for broader bandwidth per user, which results from the continuous development of new bandwidth-hungry services and applications, creates the motivation to upgrade the currently deployed Time-Division Multiplexing Passive Optical Networks (TDM-PONs) to NextGeneration Optical Access Networks (NG-OANs). Beside the need for more bandwidth per user, a further extension in the range and an increase in the split ratio are highly desirable in PONs. These additional requirements can be achieved by adopting so-called Long-Reach Optical Access Networks (LR-OANs). LR-OANs offer a promising solution that ensures a significant number of users can be supported over a longer range. Moreover, they introduce a cost-effective approach in which both the access and metro segments of the telecommunication network are combined into one backhaul segment, which results in the consolidation of many central offices into one trunk-exchange. This cost-effective approach gave us the motivation to provide a comprehensive survey on the LR-OANs. In this study, we first provide a brief review of different potential technologies, proposed for next-generation optical access. We then provide a review of different stat-of-the-art LR-OAN architectures including opportunities and challenges in each one. A comparison among them based on key network specification is also provided.
\end{abstract}

Keywords: Passive Optical Networks (PONs), Long-Reach Optical Access Network (LR-OANs), Optical Hybrid Schemes, Next-Generation Optical Access, Fiber-to-The Home FTTH, DWDM

\section{INTRODUCTION}

Gigabit-class Passive Optical Networks (gigabit class-PONs), such as Ethernet PONs (EPONs) and Gigabit PONs (GPONs), have been adopted in many countries as an alternative solution to copper-based access networks to enable the availability of broadband access services due to their huge bandwidth, which allows access users to expand their capacity to include higher bandwidth services, such as Video on Demand (VoD) and Voice over Internet Protocol (VoIP). Both EPONs and GPONs use timedivision multiplexing for the physical layer; however, they differ in terms of the data link layer protocol used, i.e., EPONs use Ethernet and GPONs use the GPON Encapsulation Method (GEM). Although gigabit class-
PONs were envisioned as an ultimate solution to the bandwidth demand problem because they offer a significantly wider bandwidth in comparison with the copper-based access networks, their capacity will be exhausted when more bandwidth-hungry services become available in the near future.

For example, High-Definition Television service (HDTV) requires on the order of $8 \mathrm{Mbps} /$ channel, making the minimum bit rate required to simultaneously support a single channel of HDTV plus data and voice equal to 10 Mbps (Shea and Mitchell, 2007a). Thus, an upgrade from the currently adopted gigabit-class PONs to the NextGeneration Optical Access Networks (NG-OANs) becomes inevitable. In addition to the need for higher bit rates, a further increase in the split ratio and an extension in the range are required in PONs to enable the support of a large

Corresponding Author: Mohamed, I.M.M., Department of Electrical, Electronics and System Engineering, UKM University, Bangi, Malaysia 
number of users over a longer range. Long-Reach Optical Access Networks (LR-OANs) are a potential candidate technology for Next-Generation Optical Access (NGOA). They provide a cost-effective solution that would reduce the capital and the operational expenditures by consolidating the number of central office sites by combining both the access and the metro segments of the telecommunication network in one extended back-haul segment. A basic telecommunication network and its simplified version, based on basic long-reach optical access network architecture, are shown in Fig. 1a and b. Much effort has been invested in creating a next-generation optical access architecture based on the long-reach optical access approach. Long-Reach Optical Access Networks (LR-OANs) were initially based on Time-Division Multiplexing (TDM) technology in which a single wavelength is shared by a significant number of Optical Network Units (ONUs) and then they were based on hybrid Time-Division Multiplexing (TDM)/Coarse Wavelength-Division Multiplexing (CWDM) and hybrid Time-Division Multiplexing (TDM)/Dense
Wavelength-Division Multiplexing (DWDM) technologies. The paper is organized as follows: Section 2 reviews the candidate technologies for nextgeneration optical access. Section 3 provides a review of the important architectures that have been proposed to create a long-reach optical access network. Section 4 summarizes the common opportunities and challenges which are in related to the proposed LR-OAN architectures. A general discussion is provided in section 5. Section 6 concludes the paper.

\section{THE POTENTIAL TECHNOLOGIES FOR NEXT-GENERATION OPTICAL ACCESS}

The next-generation optical access options, the natural result of several intensive studies, are intended to improve the performance of the currently deployed gigabit class-PONs to achieve certain goals, such as higher data rates, larger capacities and longer ranges. The following is a summary of these options.

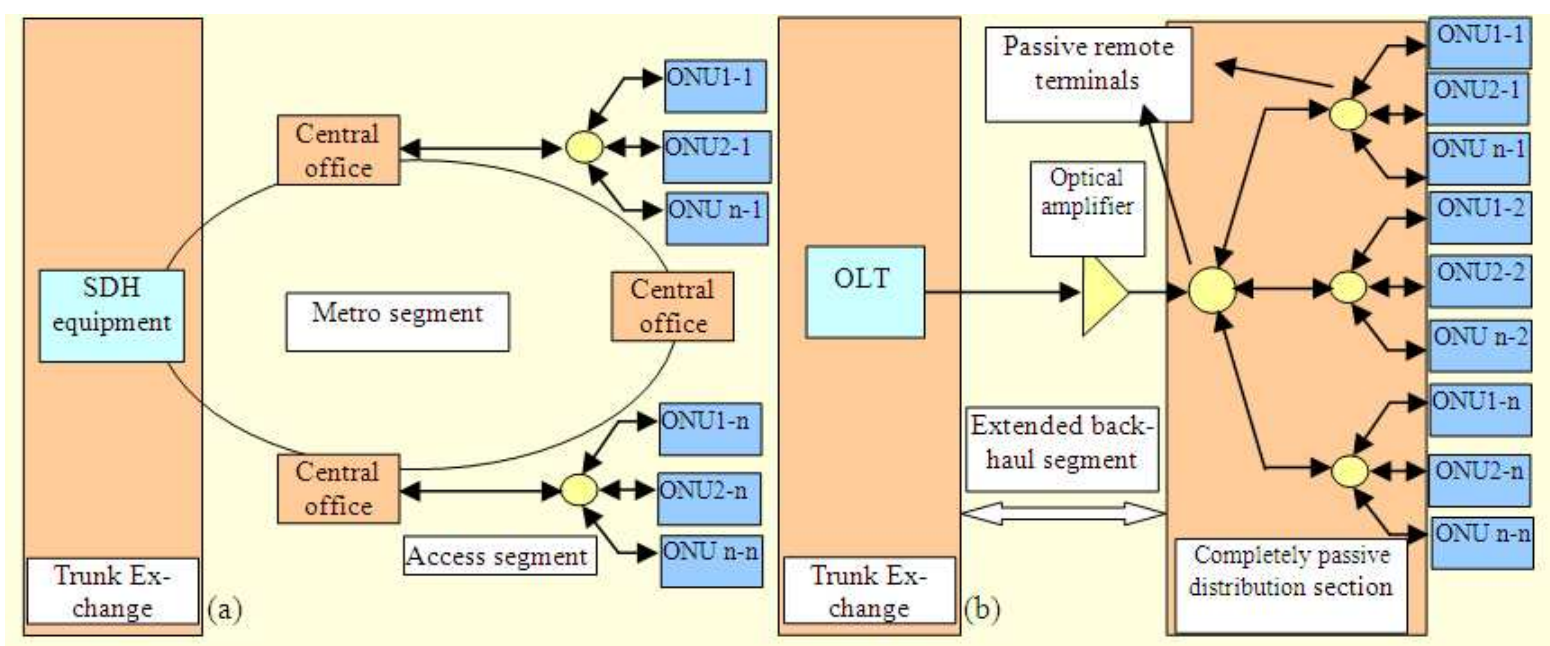

Fig. 1. (a) Metro and Access segments in a telecommunication network, (b) Simplified LR-OAN architecture

Table 1. IEEE and ITU-T standardizations for NGOA

\begin{tabular}{lllll}
\hline & 10GEPON & XG-PON & WDM GPON & LR GPON \\
\hline Standard work group & IEEE 802.3 av & ITU-T G.987 & ITU-T G.984.5 & ITU-T G.984.6 \\
Enhancement option & Line speed & Line speed & WDM & reach extension \\
Data rate US/DS (Gbps) & $10 / 10$ symmetric & $10 / 10$ symmetric & 2.488 or 10 per & 2.488 or 10 per \\
& $10 / 1$ asymmetric & $10 / 2.5$ asymmetric & $\lambda / 1.244$ per $\lambda$ & $\lambda / 1.244$ per $\lambda$ \\
Power budget (dB) & $20 / 24 / 29$ & nominal $(29-31)$ & 29 & 27.5 both span \\
& & extended (33-35) & & \\
\hline
\end{tabular}




\subsection{High-Data-Rate TDM-PON}

To rectify the significant growth in bandwidth demand that is anticipated in the near future, the Institute of Electrical and Electronics Engineers (IEEE) and the International Telecommunications Union's Telecommunication Standardization Sector (ITU-T) ratified their standards (IEEE803.av, 10GEPON and ITU-T.987, XG-PON) in 2009 and 2010, respectively (IEEE Std 802.3av, 2009; ITU-TR, 2008). Both standards were mainly developed to enhance the bit rate of the current gigabit-class PONs (EPON and GPON). 10GEPON specifies symmetric 10-Gbps transmission downstream and upstream; however, in asymmetric transmission, 10-Gbps are provided downstream and 1-Gbps are provided upstream. XG-PON also specifies symmetric 10-Gbps transmission both downstream and upstream. However, it specifies an asymmetric 10-Gbps transmission downstream and 2.5-Gbps transmission upstream. Because it is expected that any optical link will lose several $\mathrm{dBs}$ of its allowance when the bit rate is increased to $10 \mathrm{Gbps}$, both standards recommend the use of Forward Error Correction (FEC) codes. However, the use of such codes would come at the expense of throughput reduction. Table 1 summarises the key specifications of both standards.

\subsection{The Wavelength-Division Multiplexing (WDM) PON}

Wavelength-Division Multiplexing PONs (WDMPONs) were mainly proposed to exploit the large wavelength counts available in the fibre (its virtual unlimited bandwidth) to achieve a significant increase in the system capacity. WDM has an important advantage over TDM in that more privacy and security are ensured for each ONU by allocating the ONU a dedicated pair of wavelengths (virtual P2P connection). Additionally, it is a suitable candidate for optical fibre applications in which feeder fibres are not available in abundance. Moreover, WDM allows coexistence in an open access environment among different network operators; new operators can share the same Optical Distribution Network (ODN) with legacy operators. However, the main obstacle preventing the adoption of WDM-PONs in next-generation optical access is based on cost consideration. Deploying WDM would definitely increase the cost because it requires additional filters for wavelength separation purposes, thus enforcing each
ONU to handle the data traffic via a stable pair of wavelengths to ensure a successful filtering process. To reduce the cost of the WDM system, different techniques have been proposed to unify the design of the ONU (i.e., colourless ONU) (Kani, 2010). To further reduce the cost, it is recommended that Coarse Wavelength-Division Multiplexing (CWDM) be considered, in which $20 \mathrm{~nm}$ wavelength spacing is used, allowing extra tolerance for wavelength drift. A review of different WDM-PON architectures is provided in (Grobe and Elbers, 2008).

\subsection{Hybrid TDM/WDM-PON}

This solution is mainly based on the exploitation of the advantages provided by both TDM-PONs and WDM-PON. In the TDM/WDM-PON, the high data rate provided by the TDM-PONs and the large number of wavelengths offered by the WDM-PONs are joined into one hybrid. The first commercial colourless gigabit TDM/WDM-PON was proposed in (Hyun et al., 2010) for Korea Telecom. Based on the wavelength grid used, TDM/WDM-PONs can be categorised as either TDM/CWDM-PONs or TDM/DWDM-PONs. To achieve further benefits, such as load balancing, resilience and power saving, a dynamic TDM/WDMPON hybrid scheme was proposed in which dynamic wavelength assignment is used (Kani, 2010). However, adopting such a scheme would enforce the use of burst mode receivers in the downstream direction (a burst mode receiver is required in each $\mathrm{ONU}$ ) due to the frequent changes in wavelength.

\subsection{Long-Reach Optical Access (LROA)}

Long-reach optical access was initially proposed to overcome the limitations of TDM-PONs and WDMPONs, such as the low splitting ratio and limited range. LROA improves the cost sharing and the efficiency in the access system by supporting more users over a common physical medium. Moreover, it offers a costeffective solution that would further reduce the capital and the associated operational expenditures by consolidating the number of central office sites, combining both the access and metro segments of the telecommunication network in one extended back-haul segment. To extend the physical range and increase the splitting ratio, solutions based on signal amplification were required. In the LROA, deploying optical amplifiers in the field rather than using conventional repeaters was recommended such that performing complex and expensive processes, such as photon-to-electron conversion, retiming, reshaping, electrical amplification and electron- 
to-photon conversion, would be avoided. Additionally, unlike conventional repeaters, optical amplifiers are transparent to the bit-rate changes and the data format used. While the long-reach optical access option offers the aforementioned opportunities, it also introduces many new challenges. For example, the long-reach optical access networks should be able to overcome the so-called Amplified Spontaneous Emission (ASE) noise introduced by the optical amplifiers. Additionally, they should be able to support the upstream burst-mode transmission. Moreover, they should be able to allow wideband optical amplification if a Coarse WavelengthDivision Multiplexing (CWDM) grid is used. A further challenge is to decrease the propagation delay time while using the LROA. For example, a 100-km loop would lead to an increased Round-Trip Time (RTT) from 0.2 $\mathrm{ms}$ in a $20 \mathrm{~km}$ loop to $1 \mathrm{~ms}$, which would degrade the receiver sensitivity performance. Finally, because the LROA aims to support a significant number of users over a common physical medium, further issues relevant to security improvement and reliability in the optical access systems, which might not have been addressed previously, would be of significant importance. Further details regarding several important LR-OAN architectures and their enabling technologies are provided in section 3.

\subsection{Fibre Wireless Access (FiWi)}

FiWi networks are mainly developed based on a combined architecture, using both optical and wireless access networks in which the significant capacity provided by the optical network and the mobility offered by the wireless network are joined into one hybrid to enable the delivery of quad-play services (video, data, voice and mobility) (Ghazisaidi and Maier, 2011). Generally, FiWi networks can be classified as Radioover-Fibre networks (RoF) or Radio and Fibre networks ( $\mathrm{R}$ and $\mathrm{F}$ ). In RoF networks, an optical carrier is modulated by a Radio Frequency (RF) signal in a central office before being sent over an optical fibre to a Remote Antenna Unit (RAU) in which it is sent again over air. Although RoF networks offer a cost-effective solution because the signals are processed in a centralised location, they impose undesirable additional propagation delay to users who communicate solely in a wireless medium, which limits the performance. To overcome this limitation, an approach based on the emerging discrete optical and wireless networks, each with its own Medium Access Control (MAC) protocol, into one single integrated network was proposed. This approach led to Radio and Fibre networks ( $\mathrm{R}$ and $\mathrm{F}$ networks). In $\mathrm{R}$ and F networks, the wireless users can communicate directly through their medium rather than being propagated over the optical network. Moreover, the system resilience is increased because the wireless traffic can be maintained even when the connectivity with the optical part of the network is lost. A survey of FiWi architectures can be found in (Tsagklas and Pavlidou, 2011).

\subsection{Advanced Modulation Techniques (OCDMA and OOFDM)}

Due to the continuous and ever-increasing demand for higher data rates over the dispersive optical medium, PONs based on deploying an advanced modulation technique, such as Optical Code-Division Multiple Access (OCDMA) or Optical Orthogonal FrequencyDivision Multiplexing (OOFDM), will be of significant importance in the near future because these techniques show enhanced resistance to the chromatic dispersion effect. The technologies also add a new dimension in which a suppressed dynamic-range PON can be achieved. Moreover, they lead to increased spectral efficiency, which in turn increases the capacity in the PONs. While the details of these techniques are not provided in this study, reviews can be found in (Kamijoh et al., 2009; Chang et al., 2009).

\section{LONG-REACH OPTICAL ACCESS NETWORKS (LR-OANS)}

Long-Reach Optical Access Networks (LR-OANs) were essentially proposed to realise certain objectives that could not be achieved previously in the TDMPONs and the WDM-PONs, such as a higher splitting ratio $(>64)$ and longer range $(>20 \mathrm{~km})$. Initially, they were proposed based on the legacy TDM-PONs in which a single wavelength is shared among a significant number of ONUs; later, they were proposed based on hybrid TDM/CWDM- and TDM/DWDM-PONs.

\subsection{TDM-Based Long-Reach Optical Access Networks (TDM-LROANs)}

The Photonic Local Access Network Project (PLANET) was initiated in the mid-1990s to develop a cost-effective and full-service access network, called a super passive optical network (SuperPON). The project was mainly based on a potential upgrade to the G.983 architecture (broadband PON, BPON), including an increased split ratio (2048), longer range $(100 \mathrm{~km})$ and 
higher bit rates $(2.5$ Gbps downstream and $311 \mathrm{Mbps}$ upstream) (Van De Voorde et al., 2000). To increase the splitting ratio and extend the range, optical amplifiers were used in both the downstream and the upstream directions. At first, the maximum splitting ratio achieved was 1024 , which is half of that targeted by the project. To further increase the splitting ratio, a technique based on incorporating the optical amplifiers in parallel in the distribution section was used. Although a split ratio of 2048 was achieved by using this technique, applying a complex gating protocol was necessary to overcome the detrimental effect of noise funnelling, which is the result of the parallel placement of the optical amplifiers in the distribution section. As shown in Fig. 2, the SuperPON was based on three stages of optical amplification.

The first stage was intended to extend the range and the final two stages were assigned to increase the split ratio. While EDFAs were used in SuperPONs for optical amplification in the downstream direction because of their low noise, low cross talk, high gain and high bandwidth, SOAs were used in the upstream direction due to their capability to support the faster burst-mode transmission. However, they introduced more noise. The architecture shown in Fig. 3 is the long-reach PON.

It was developed for British Telecom and was intended to satisfy the anticipated future growth in bandwidth demand and to consolidate the number of central office sites throughout the country (Shea and Mitchell, 2007b). As illustrated in Fig. 3, the attenuated optical signal is received at the local exchange site, where it is subjected to dual-stage optical amplification to overcome the next attenuation, which is imposed by the backhaul section. Because the backhaul attenuation in this architecture was relatively large (on the order of $37 \mathrm{~dB}$ ), a pre-amplifier was incorporated to increase the receiver sensitivity. To further improve the receiver sensitivity, a $2 \mathrm{~nm}$ band optical filter was incorporated prior to the receiver. This helped to reduce the Amplified Spontaneous Emission (ASE) noise, achieving the targeted BER of 10e-10. However, incorporating such a narrow band optical filter reduces the tolerated limit for wavelength drift. This reduction in the wavelength drift tolerance imposes an additional requirement at the transmitter side to ensure precise wavelength production, which further increases the cost. To gain extra margin, a Forward Error Correction code (FEC) was assigned. The system was able to support a symmetric 10 -Gbps rate for both the downstream and the upstream traffic over a $100-\mathrm{km}$ backhaul fibre. Although the system has a split ratio of 1024 , which is half of that achieved in SuperPON, its distribution section is completely passive and does not require the aforementioned gating protocol, as required in SuperPON.

\subsection{TDM/CWDM-Based Long-Reach Optical Access Networks (TDM/CWDM-LR-OANs)}

In Coarse Wavelength-Division Multiplexing (CWDM), a $20 \mathrm{~nm}$ wavelength spacing is used, allowing simultaneous transmission over an optical fibre such that the number of transmitted wavelengths is fewer than that specified in Dense Wavelength-Division Multiplexing (DWDM) but more than that specified in standard Wavelength-Division Multiplexing (WDM). This relatively large spacing offers increased tolerance for wavelength drift, allowing the use of low-cost, uncooled lasers. A hybrid TDM/CWDM-LR-OAN was proposed in (Iannone et al., 2008). The architecture shown in Fig. 4 enables simultaneous transmission in which four TDM-PONs were combined using a CWDM grid for sending over a $60-\mathrm{km}$ shared backhaul fibre. Using this architecture, a wavelength range from 1490 to $1550 \mathrm{~nm}$ was allocated for the downstream transmission, while a wavelength range from 1290 to $1350 \mathrm{~nm}$ was allocated for the upstream transmission.

The main challenge in this configuration was the necessity to perform wideband optical amplification due to the relatively large CWDM grid used. For example, a typical 4-channel CWDM system requires a bandwidth of $70 \mathrm{~nm}$. To this end, an SOA-Raman hybrid amplifier was used for signal amplification in both directions. An SOA-Raman hybrid amplifier that offers a wider bandwidth than the standalone SOA amplifier was proposed in (Iannone et al., 2006; Lee et al., 2008). The architecture was able to support up to 128 users by incorporating a remote node unit RNU, which contained two SOA-Raman hybrid amplifiers and two CWDM couplers followed by four $(2 \times 2)$ splitters and two $(1 \times 16)$ splitters, as shown in Fig. 4.

\subsection{TDM/DWDM-Based Long-Reach Optical Access Networks (TDM/DWDM-LR-OANs)}

Because dense wavelength-division multiplexing (DWDM) relies on narrowband optical filtering $(50 \mathrm{GHz}$ or $100 \mathrm{GHz}$ ), one of the main challenges in deploying TDM/DWDM-based long-reach optical access networks (TDM/DWDM-LR-OANs) was to choose a suitable and cost-effective transmitter technology that can ensure precise wavelength production within the DWDM grid. To avoid the potential costs associated with the use of wavelength-specific lasers in the ONU, the hybrid 
TDM/DWDM-LR-OAN proposed in (Talli and Townsend, 2006) was based on the employment of a centralised optical carrier distribution and wavelengthindependent remote modulation schemes. The transmitter was based on the use of an Electro-Absorption Modulator (EAM) that was integrated with two semiconductor optical amplifier SOAs. The centralised optical carrier received at the ONU input was amplified by the first SOA, modulated by the EAM modulator, amplified again by the second SOA and then transmitted over the fibre. The C-band was separated by a $5 \mathrm{~nm}$ guard band and then was allocated for both directions such that the wavelength range from 1529 to $1541.6 \mathrm{~nm}$ was allocated for the downstream traffic, whereas the wavelength range from 1547.2 to $1560.1 \mathrm{~nm}$ was allocated for the upstream traffic. A standard $1 \times 40$ channel Arrayed Waveguide Grating (AWG) was used. Channels 1-17 were assigned to handle the downstream traffic, channels 24-40 were assigned to handle the upstream traffic and channels $18-23$ were designated as the guard band channels. This channel assignment gave the opportunity to combine 17 TDM-PONs, each located $12 \mathrm{~km}$ apart from the local exchange by using the DWDM grid $(0.8 \mathrm{~nm})$. In addition to the optical amplification process at the local exchange site, the simultaneous transmission over an extra $88 \mathrm{~km}$ backhaul fibre was enabled, resulting in the possibility for transmission over a total of $100 \mathrm{~km}$, as shown in Fig. 5.

Because a high data rate was targeted in this architecture (10 Gbps), the dispersion effect was not negligible; thus, a resulting power penalty was expected. To reduce this power penalty, a length of DispersionCompensating Fibre (DCF) was incorporated at the core exchange to be shared by both the upstream and downstream traffic before being separated using a circulator. The system was able to support a symmetric $10 \mathrm{Gbps}$ rate and to accommodate up to 4352 users (17 PONs with a split ratio of 256 for each). The long-reach optical access architecture proposed in (Shea and Mitchell, 2007c) was based on a wavelength conversion technique, i.e., rather than using a wavelength-specific laser in the ONU, a wavelength converter is inserted into the distribution fibre to convert the unstable wavelength that is produced by the ONU to a stable DWDM wavelength before transmission over the backhaul fibre. This technique led to further reductions in cost because cooling equipment was not required in the ONU.

Figure 6 shows the upstream architecture of the longreach wavelength-converted PON. The distribution section was based on a standard GPON infrastructure in which a 20 $\mathrm{km}$ single-mode fibre and size 64 splitter are used. The wavelength converting process was achieved by using a simple cross-gain modulation wavelength converter. In this process, an Erbium-Doped Fibre Amplifier (EDFA) was used as a pre-amplifier to saturate the data signal (pump signal) before the signal sent to the second cascaded SOA, where a continuous-wavelength laser (probe wavelength) was injected in the opposite direction. The converted wavelength, (modulated probe wavelength), was then multiplexed into a $100 \mathrm{~km}$ single-mode fibre (a backhaul fibre) through an AWG. By using a 20-channel AWG with $100 \mathrm{GHz}$ frequency spacing, the long-reach wavelength-converted PON was able to support 1280 users over $120 \mathrm{~km}$ with a minimum data rate of 38.8 Mbps for each. Proposed a hybrid DWDM/TDM- longreach OAN in (Jung et al., 2012). The architecture shown in Fig. 7 was based on using a bismuth-based bidirectional EDFA. The Bi-EDFA was located a $50 \mathrm{~km}$ apart from the OLT. Similar to the proposed hybrid DWDM/TDM in (Talli and Townsend, 2006), a DCF was located at the OLT to compensate for the dispersioninduced signal distortion in the fiber transmission link. The architecture was able to allow for bidirectional transmission at a line rate of a $10 \mathrm{Gbps}$ over $75 \mathrm{~km}$ reach with 8 split users. While the reach and the split size was limited by the insufficient saturated WDM signal gain of the Bi-EDFA, a theoretical investigation in that study showed that a further increase in the reach and/or split can be obtained by optimizing the Bi-EDF length and pumping conditions. Mohammad Syuhaimi and Ibrahim Mohamed proposed a hybrid Time-Division Multiplexing (TDM)/Dense Wavelength-Division Multiplexing (DWDM) scheme in (Syuhaimi et al., 2013) to implement a cost-effective and scalable LongReach Optical Access Network (LR-OAN). The proposed architecture shown in Fig. 8 was mainly based on the involvement of an Arrayed Waveguide Grating (AWG) and an Erbium-Doped Fibre Amplifier (EDFA) in one configuration and the exploitation of their combined characteristics. The AWG was employed to increase the fibre plant utilisation, provide coexistence among different service providers through its Wavelength Cyclic Property (WCP) and to enable handling of the downstream and upstream traffic via the same input/output ports by exploiting the system's Free Spectral Range Property (FSRP). According to the Wavelength Cyclic Property (WCP), if a number $m$ of identical aggregations $(\lambda 1-\lambda n)$, transmitted by m OLTs, enter $\mathrm{N}$ input ports of an $\mathrm{N} \times \mathrm{N} A W G$, in which $\mathrm{m}=\mathrm{n}=$ 
$\mathrm{N}$, then the $\mathrm{m}$ aggregations will be distributed among the output ports of the $\mathrm{N} \times \mathrm{N}$ AWG in a non-overlapping manner. Each output port carries a replica of the same frequency range, although from different OLTs. According to the Free Spectral Range Property (FSRP), if two wavelengths enter the same input port of the $\mathrm{N} \times \mathrm{N}$ AWG, then they will emerge from the same output port with a separation determined by its periodic frequency range Free Spectral Range (FSR) (Kaneko et al., 1999).

Both the WCP and FSRP were targeted in this architecture to achieve frequency re-use and to allow handling of upstream and downstream traffic via the same input and output ports. The EDFA was employed to extend the reach and to increase the splitting ratio. In addition to its role in extending the reach and increasing the splitting ratio, the EDFA added a new dimension, allowing a simpler and more evolved network in which OLTs were divided into groups. Here, the number of OLT groups is $\mathrm{Ng}=\mathrm{N} / \mathrm{m}$, where $\mathrm{N}$ represents the number of the AWG input ports and $m$ represents the number of the OLTs in one group; accordingly, an aggregation of $\lambda 1-\lambda n$ was considered for each OLT, where $n=N$.

In this innovative architecture, service can be provided through $100 \mathrm{~km}$ backhaul fibres to $\mathrm{N}$ regions.
Each region can provide service for up to $\mathrm{N} / \mathrm{m}$ zones with different bit rates for each provided through feeder fibres, used with either a multi-fibre or a single-fibre approach. In the multi-fibre approach, a $100 \mathrm{~km} \mathrm{SM}$ fibre was assigned for each region (the backhaul fibre), while several $20 \mathrm{~km}$ SM fibres, equivalent in number to the number of OLTs in one group, was assigned for each zone (the feeder fibres); each fibre provides different bitrate service. A two-stage power splitter (1:4) and (1:64), giving a total split size of $(1: 256)$, was connected at the end of each feeder fibre. In the single-fibre approach, service is provided to each zone via a common fibre by using $(\mathrm{N} / \mathrm{m}) \times 1$ WDM-Mux and $1 \times(\mathrm{N} / \mathrm{m})$ WDMDemux. Although the single-fibre approach offers a more efficient solution because service is provided to each zone through a common fibre, it nonetheless imposes additional losses, which necessitate shortening the feeder fibre. By considering a $15 \times 15$ AWG and 3 OLTs in each OLT group $(m=3)$, the architecture was able to accommodate up to 768 ONUs $(3 \times 256)$ for each zone, a total of 3840 ONUs for each region, resulting in an overall system capacity of 57600 ONUs for the 15 regions. Table 2 provides a comparison among the aforementioned architectures, based on the key network specifications.

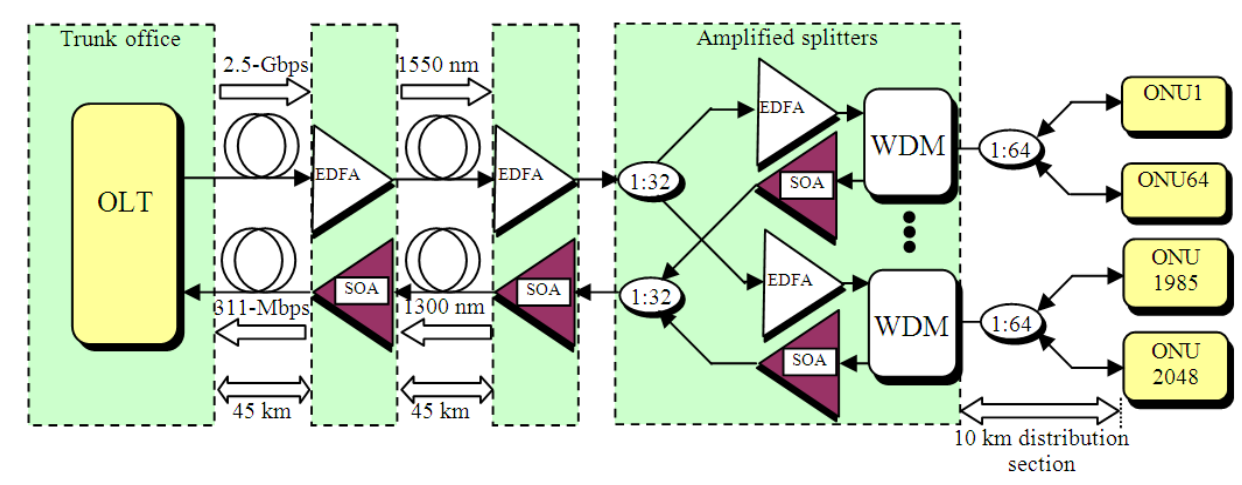

Fig. 2. The SuperPON architecture

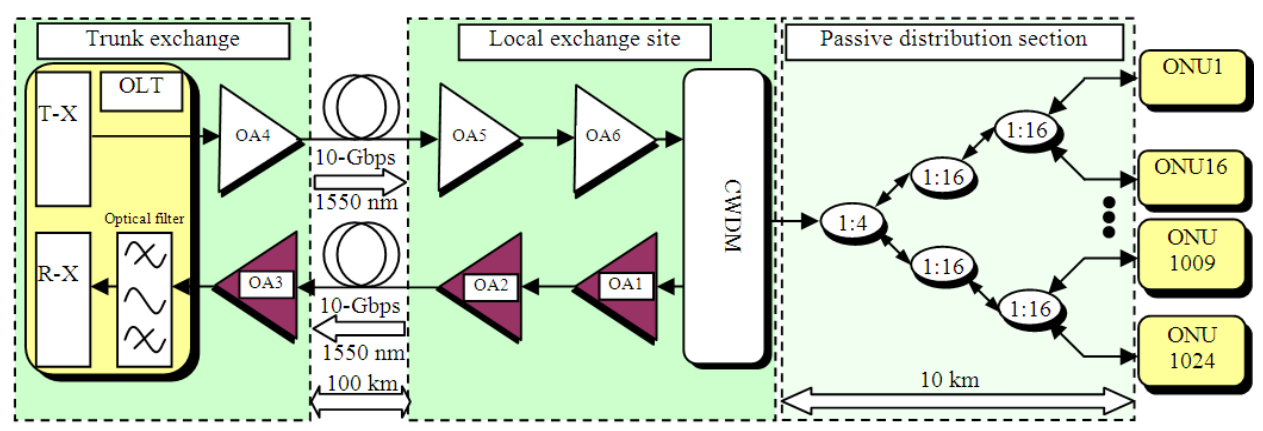

Fig. 3. The Long-reach PON architecture 


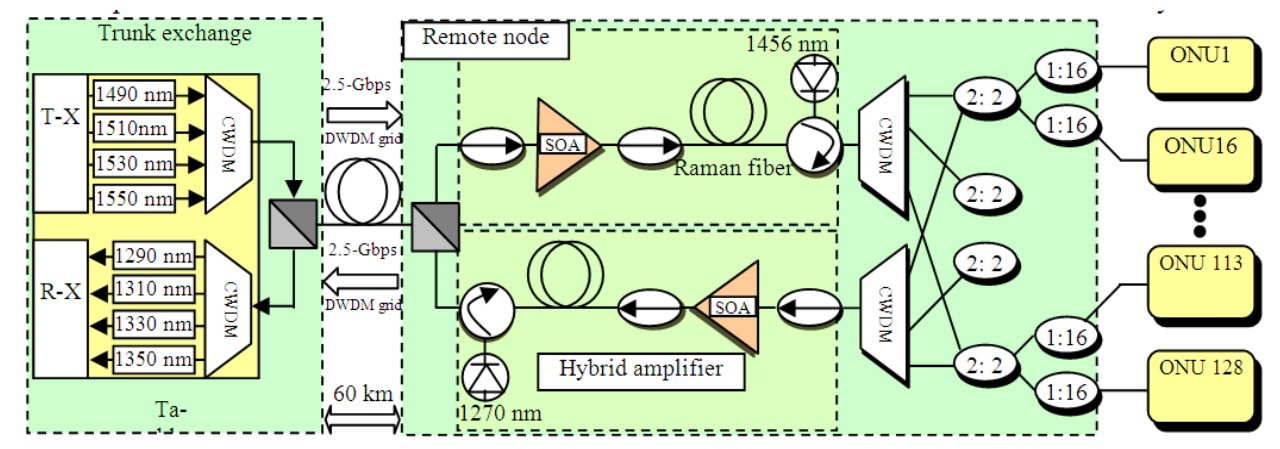

Fig. 4. The Hybrid TDM/CWDM architecture

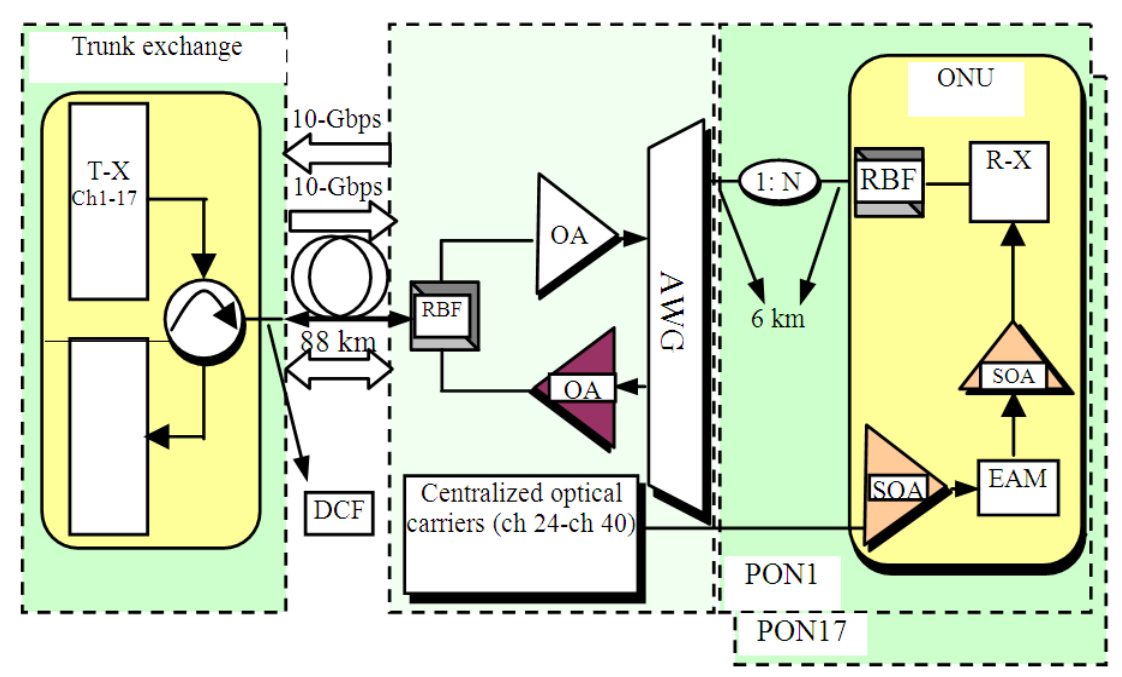

Fig. 5. Hybrid TDM/DWDM-LR-OAN architecture

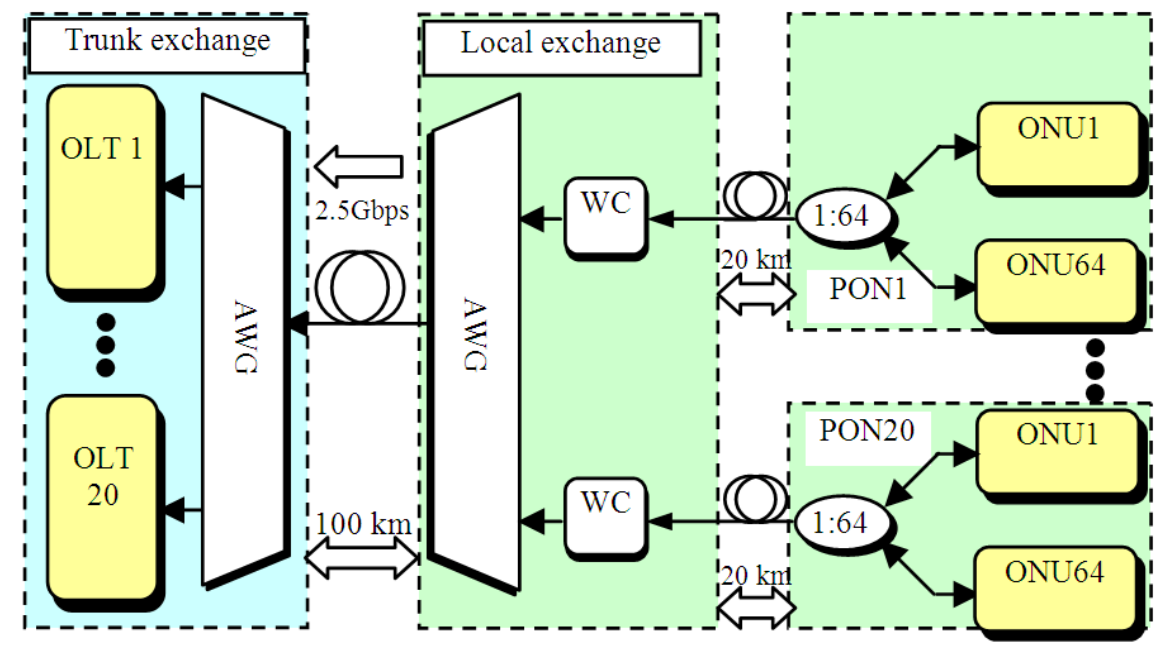

Fig. 6. WC-Based LR-OAN architecture 


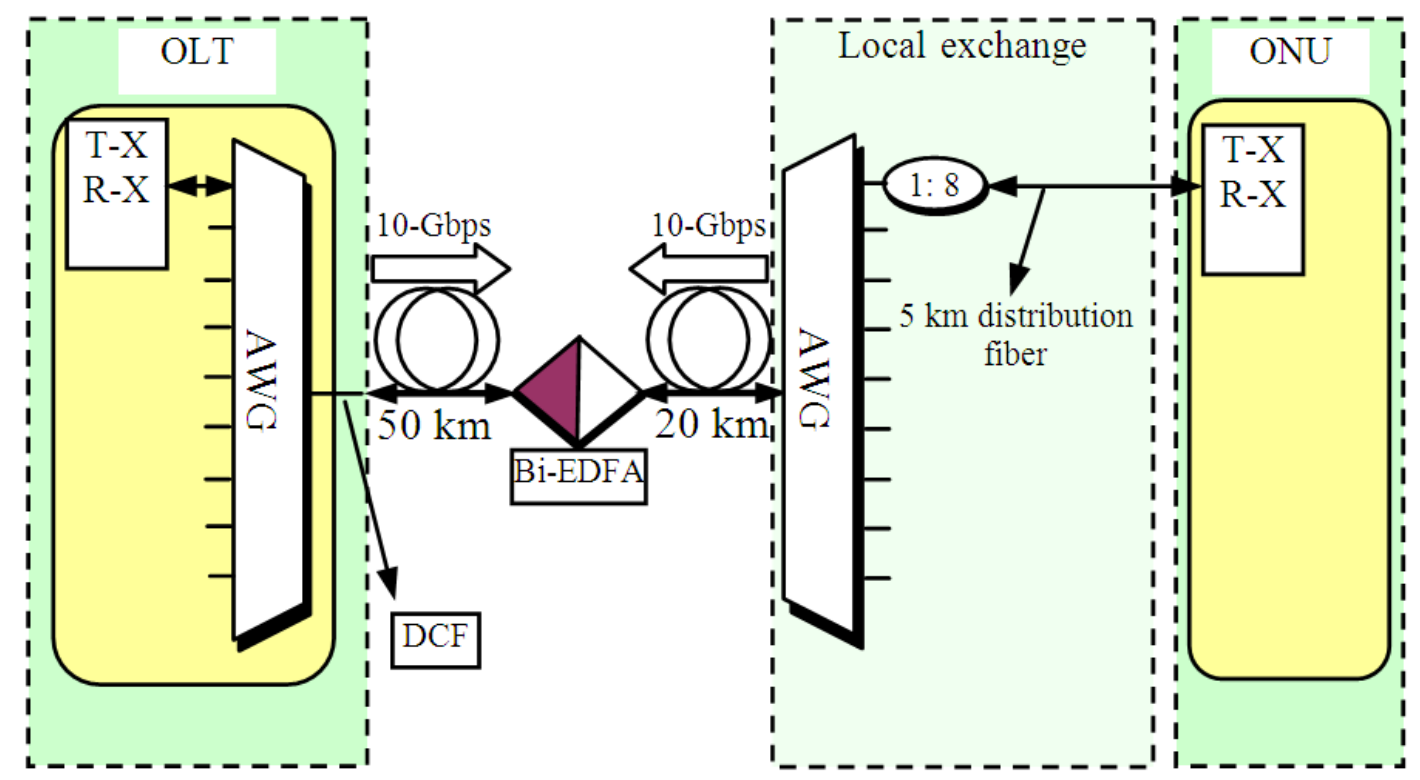

Fig. 7. Bi directional bismuth- based EDFA LR-OAN architecture

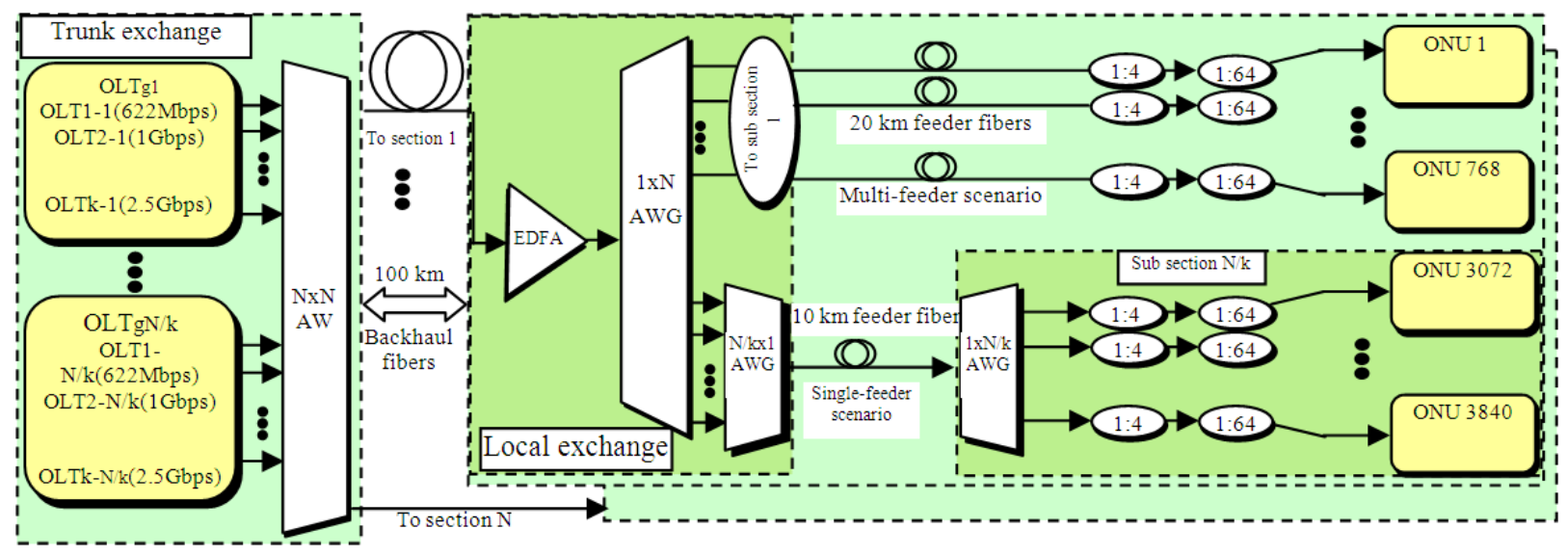

Fig. 8. The AWG and EDFA-Based LR-OAN architecture

Table 2. LR-OANs architectures

\begin{tabular}{|c|c|c|c|c|c|c|c|}
\hline Classification & $\begin{array}{l}\text { SuperPON } \\
\text { architecture } \\
\text { TDM-LR-OAN }\end{array}$ & $\begin{array}{l}\text { Long-reach } \\
\text { PON archi } \\
\text { tecture } \\
\text { TDM-LR-OAN }\end{array}$ & $\begin{array}{l}\text { SOA-raman } \\
\text { hybrid archi } \\
\text {-tecture } \\
\text { TDM/DWDM- } \\
\text { LR-OAN }\end{array}$ & $\begin{array}{l}\text { Centralized } \\
\text { optical carrier } \\
\text { architecture } \\
\text { TDM/DWDM- } \\
\text { LR-OAN }\end{array}$ & $\begin{array}{l}\text { Wavelength } \\
\text { converted PON } \\
\text { architec-ture } \\
\text { TDM/DWDM- } \\
\text { LR-OAN }\end{array}$ & $\begin{array}{l}\text { Bi directional } \\
\text { bismuth-based } \\
\text { EDFA archi-tecture } \\
\text { TDM/DWDM- } \\
\text { LR-OAN }\end{array}$ & $\begin{array}{l}\text { AWG and } \\
\text { EDFA-bases } \\
\text { architecture } \\
\text { TDM/DWDM- } \\
\text { LR-OAN }\end{array}$ \\
\hline Accommodated users & 2048 & 1024 & 128 & 4352 & 1280 & 8/splitter & 3840/region \\
\hline Physical range (km) & 100 & 110 & 60 & 100 & 120 & 75 & 120 \\
\hline Bit rate & $2.5 \mathrm{Gbps}$ & $10 \mathrm{Gbps}$ & $2.5 \mathrm{Gbps}$ & $10 \mathrm{Gbps}$ & $2.5 \mathrm{Gbps}$ & $10 \mathrm{Gbps}$ & $\begin{array}{l}622 \mathrm{Mbps} \text {, } \\
1 \mathrm{Gbps} \text { and } 2.5 \mathrm{Gbps}\end{array}$ \\
\hline Wavelengths (nm) & $\begin{array}{l}1550 \text { (DS) } \\
1300 \text { (US) }\end{array}$ & 1550DS/US & CWDM-grid & DWDM-grid & DWDM-grid & DWDM-grid & DWDM-grid \\
\hline
\end{tabular}




\section{OPPORTUNITIES AND CHALLENGES IN LR-OANS}

Following is a summary of the common opportunities offered by the LR-OANs: The LR-OANs improve the cost-sharing and the efficiency in the access system by supporting large number of users over a common physical medium. Moreover, they offer a cost-effective solution in which the number of the central office sites is consolidated by combining both the access and the metro segments of the telecommunication network in one extended back-haul segment. In the LR-OANs, optical amplifiers are used instead of the conventional repeaters, which avoid performing complex and expensive processes, such as photon-to-electron conversion, retiming, reshaping, electrical amplification and electron-to-photon conversion. Additionally, unlike conventional repeaters, optical amplifiers are transparent to the bit rate changes and the data format used. While the LR-OANs offer the aforementioned opportunities, they introduce the following challenges: The long-reach optical access networks should be able to overcome the so called Amplified Spontaneous Emission (ASE) noise introduced by the optical amplifiers. Additionally, they should be able to support the upstream burs-mode transmission. Moreover, they should be able to allow wideband optical amplification if Coarse Wavelength Division Multiplexing (CWDM) grid is used. A further challenge in the long-reach optical access networks is to decrease the propagation delay time. For example, a 100$\mathrm{km}$ loop would lead to an increased the Round Trip Time (RTT) from $0.2 \mathrm{msec}$ in a $20 \mathrm{~km}$ loop to $1 \mathrm{msec}$, which would degrade the receiver sensitivity performance. Finally, because the LROA aims to support a large number of users over a common physical medium, further issues relevant to security improvement and reliability in the optical access systems, which might not have been addressed previously, would be of great importance. In this context, several schemes were proposed in the literature to increase the level of survivability in FTTH networks (Ab-Rahman et al., 2011; Ab-Rahman et al., 2012)

\section{DISCUSSION}

The various requirements in the NGOA, such as the need to have a higher bit rate, extended reach, increased splitting ratios and mobility, provided the researchers with the opportunity to propose different architectures.
This study provides a review of these architectures including contributions and challenges of each. A comparison among them based on key network specifications is also provided. The paper begins by introduce and compare the already standardized PONs for NGOA (IEEE 802.3av [10G-EPON] and ITU-T G.987 [XG-PON]) (IEEE Std 802.3av, 2009; ITU-TR, 2008).

It then proceeds to provide a review on the standardization activities for NGOA adoption. Several options including the WDM-PONs (Kani, 2010; Grobe and Elbers, 2008), hybrid TDM/WDM-PONs (Hyun et al., 2010), FiWi (Ghazisaidi and Maier, 2011; Tsagklas and Pavlidou, 2011), Advanced Modulation Techniques (AMTs) (Kamijoh et al., 2009; Chang et al., 2009), TDM-Based LR-OANs (Van de Voorde et al., 2000; Shea and Mitchell, 2007b), TDM/CWDM-Based LR-OANs (Iannone et al., 2008) and TDM/DWDM-Based LR-OANs (Talli and Townsend, 2006; Shea and Mitchell, 2007c; Jung et al., 2012; Syuhaimi et al., 2013) were reviewed. Although many of these proposed architectures are interesting from the technical perspective, the cost, bandwidth per user and the number of accommodated subscribers remain the dominant factors for commercial success. Among all optical access technologies, proposed for NGOA, the LR-OAN, is a very promising candidate due to its cost-effective approach in which the number of central office sites is consolidated by combining both the access and metro segmrnts of the telecommunication network in one bachhaul segment.

\section{CONCLUSION}

A review of the various potential candidate technologies for next-generation optical access was provided. Among all technologies proposed for nextgeneration optical access is the long-reach optical access technology. A review of different stat-of-the-art longreach optical access architectures including opportunities and challenges of each one was provided. We conclude that the long-reach optical access technology will attract a significant deal of attention because of its cost-effective solution that reduces the overall cost by combining both the access and metro segments of the telecommunication network into one extended backhaul segment, which ensures that a significant number of end users can be connected directly to the core network without the need for conversion through intermediate switching stages. Although the approach of Long-Reach Optical Access (LROA) seems to be quite simple at first glance because 
it is mainly based on employing optical amplifiers in the field rather than using conventional repeaters, nonetheless, it introduces new challenges because incorporating such amplifiers is not straightforward. A discussion concerning the limitations of this technology was provided in section 4, which paved the way for future research plans.

\section{REFERENCES}

Ab-Rahman, M.S. and S.R.A. Mahir, 2011. Development of an algorithm for fiber-to-the-home passive optical network automatic self-restoration scheme using access control system. J. Comput. Sci., 7 : 1846-1853. DOI: 10.3844/jcssp.2011.1846.1853

Ab-Rahman, M.S., S.A.C. Aziz and K. Jumari, 2012. Linear protection schemes analysis in scattered placement fiber-to-the home-passive optical network using customer access protection unit solution. J. Comput. Sci., 8: 41-47. DOI: 10.3844/jcssp. 2012.41 .47

Chang, G.K., C.A. Zhensheng, J. Hung-Chang, C. MingFang and H.J. Yu et al., 2009. Key technologies of WDM-PON for future converged optical broadband access networks [invited]. IEEE J. Opt. Commun. Netw., 1: 35-50. DOI: 10.1364/JOCN.1.000C35

Ghazisaidi, N. and M. Maier, 2011. Fiber-wireless (FiWi) access networks: Challenges and opportunities. IEEE Netw., 25: 36-42. DOI: 10.1109/MNET.2011.5687951

Grobe, K. and J.P. Elbers, 2008. PON in adolescence: From TDMA to WDM-PON. Commun. Magazine IEEE, 46: 26-34. DOI: 10.1109/MCOM.2008.4427227

Hyun, J.L., S.H. Cho, H.H. Lee, E.S. Jung and J.H. Yu et al., 2010. First commercial deployment of a colorless gigabit WDM/TDM Hybrid PON system using remote protocol terminator. J. Lightw. Technol., 28: 344-351. DOI: 10.1109/JLT.2009.2037979

Iannone, P.P., H.H. Lee, K.C. Reichmann, X. Zhou and M. Du et al., 2008. Four extended-reach TDM PONs sharing a bidirectional hybrid CWDM amplifier. J. Lightw. Technol., 26: 138-143. DOI: 10.1109/JLT.2007.913072

Iannone, P.P., K.C. Reichmann, X. Zhou and S. Gray, 2006. A Discrete SOA-Raman Hybrid Amplifier with 80-nm Bandwidth. Proceedings of the European Conference on Optical Communications, Sept. 24-28, IEEE Xplore Press, Cannes, France, pp: 1-2. DOI: 10.1109/ECOC.2006.4800967
IEEE Std 802.3av, 2009. Physical layer specifica-tions and management parameters for $10 \mathrm{~Gb} / \mathrm{s}$ pas-sive optical networks.

ITU-TR, 2008. 10-Gigabit-capable Passive Optical Network (XG-PON) systems. ITU-T Publications.

Jung, M., Y.M. Chang and J.H. Lee, 2012. A bandseparated, bidirectional amplifier based on erbium-doped bismuth fiber for long-reach hybrid DWDM-TDM passive optical networks. J. Optical Commun. Netw., 4: 165-172. DOI: 10.1364/JOCN.4.000165

Kamijoh, T., M. Kashima, H. Tamai, M. Sarashina and H. Iwamura et al., 2009. CDM-technologies for next generation optical access networks. Proceedings of the Conference on Optical Fiber Communication incudes post deadline papers, Mar. 22-26, IEEE Xplore Press, San Diego, CA, pp: 1-3.

Kaneko, A., T. Goh, H. Yamada, T. Tanaka and L. Ogawa et al., 1999. Design and applications of silica-based planar lightwave circuits. IEEE J. Selected Top. Quantum Electron., 5: 1227-1236. DOI: $10.1109 / 2944.806745$

Kani, J.I., 2010. Enabling technologies for future scalable and flexible WDM-PON and WDM/TDMPON systems. IEEE J. Selected Top. Quantum Electron., 16 : 1290-1297. DOI: 10.1109/JSTQE.2009.2035640

Lee, H., P. Iannone, K. Reichmann, J. Lee and B. Palsdottir, 2008. A C/L-band gain-clamped SOARaman hybrid amplifier for CWDM access networks. IEEE Photon. Technol. Lett., 20: 196-198. DOI: 10.1109/LPT.2007.913234

Shea, D.P. and I.E. Mitchell, 2007a. Long-reach optical access technologies. IEEE Netw., 21: 5-11. DOI: 10.1109/MNET.2007.4305170

Shea, D.P. and J.E. Mitchell, 2007b. A 10-Gb/s 1024way-split 100-km long-reach optical-access network. J. Lightw. Technol., 25: pp: 685-693. DOI: 10.1109/JLT.2006.889667

Shea, D.P. and J.E. Mitchell, 2007c. Experimental upstream demonstration of a long reach wavelengthconverting PON with DWDM backhaul. Proceedings of the Conference on Optical Fiber Communication and the National Fiber Optic Engineers Conference, Mar. 25-29, IEEE Xplore Press, Anaheim, CA., pp: 1-3. DOI: 10.1109/OFC.2007.4348900 
Syuhaimi, M., B. Ab-Rahman and I.M.M. Mohamed, 2013. Highly utilized fiber plant with extended reach and high splitting ratio based on AWG and EDFA characteristics. Etri J., 35: 786-796. DOI: 10.4218/etrij.13.0112.0793

Talli, G. and P.D. Townsend, 2006. Hybrid DWDMTDM long-reach PON for next-generation optical access. J. Lightw. Technol., 24: 2827-2834. DOI: 10.1109/JLT.2006.875952
Tsagklas, T. and F.N. Pavlidou, 2011. A survey on radio-and-fiber FiWi network architectures. J. Selected Areas Telecommun.

Van de Voorde, C.M., J.M. Vandewege and X.Z. Qiu, 2000. The superPON demonstrator: An exploration of possible evolution paths for optical access networks. IEEE Commun. Mag., 38: 74-82. DOI: $10.1109 / 35.819899$ 